\title{
Non-Clinical Premating Trial Phase
}

National Cancer Institute

\section{Source}

National Cancer Institute. Non-Clinical Premating Trial Phase. NCI Thesaurus. Code C124606.

The period of time in a non-clinical study prior to cohabitation. 